\title{
Oral decontamination with chlorhexidine reduced ventilator associated pneumonia in patients needing mechanical ventilation for $\geqslant 48$ hours
}

Koeman $M$, van der Ven AJ, Hak E, et al. Oral decontamination with chlorhexidine reduces the incidence of ventilator-associated pneumonia. Am J Respir Crit Care Med 2006;173:1348-55.

$Q$ In patients requiring mechanical ventilation for $\geqslant 48$ hours, is oral decontamination with chlorhexidine $(\mathrm{CHX})$ or $\mathrm{CHX}$ plus colistin (COL) effective for reducing ventilator associated pneumonia (VAP)?

\section{METHODS}

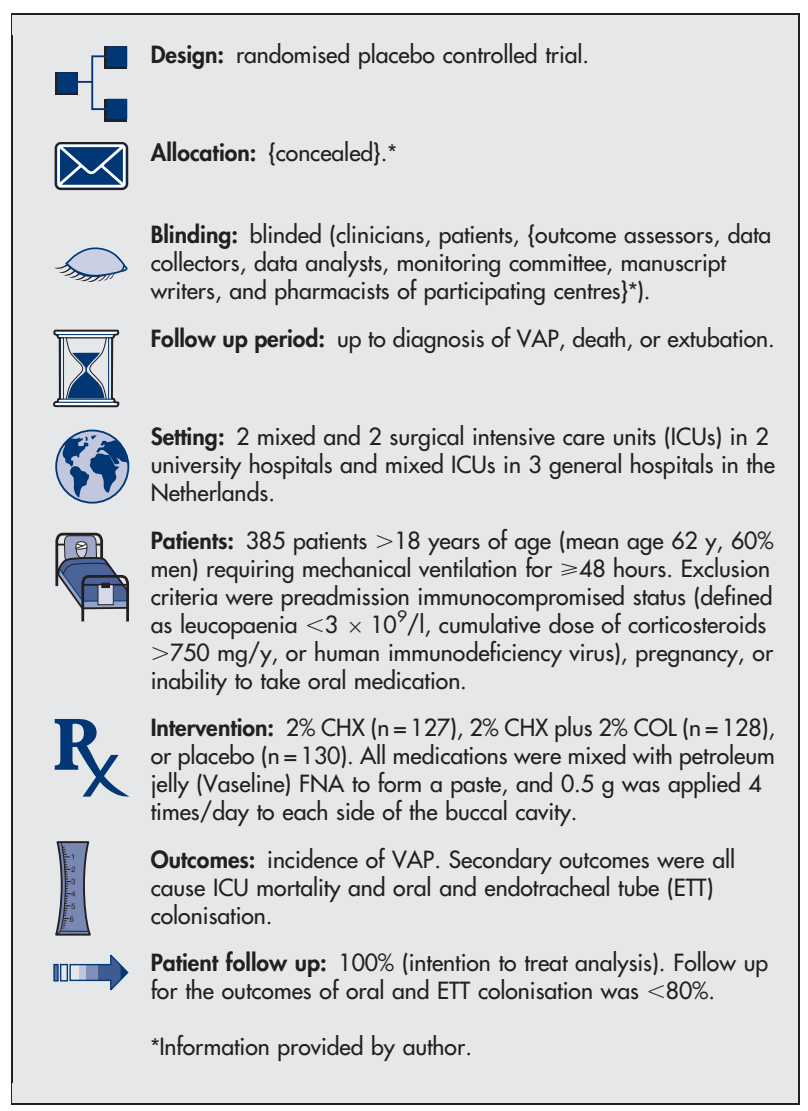

\section{MAIN RESULTS}

The CHX and CHX plus COL groups had lower incidences of VAP than the placebo group (table). The CHX and CHX plus COL groups did not differ from placebo for all cause ICU mortality (hazard ratio

For correspondence: Dr $M$ M Koeman, University Medical Center Ütrecht, Utrecht, The Netherlands. groenendijk-koeman@wanadoo.nl

Source of funding: Netherlands Organization for Health Research and Development.
$1.12,95 \%$ CI 0.72 to 1.17 and 1.02 , CI 0.66 to 1.59 , respectively). Results for oral and ETT colonisation are not reported here because $<80 \%$ of patients were included in the analysis.

\section{CONCLUSION}

In patients requiring mechanical ventilation for $\geqslant 48$ hours, oral decontamination with chlorhexidine reduced ventilator associated pneumonia.

\section{A modified version of this abstract appears in ACP Journal Club.}

\section{Commentary}

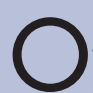
ropharyngeal colonisation with potential pathogens is central in the pathogenesis of VAP. Preventing VAP using oral decontamination with non-absorbable antibiotics is not widespread because of concerns about emergence of antibiotic resistance. Koeman et al used orally applied CHX or CHX plus $\mathrm{COL}$ as alternative agents and found both treatments to be effective in VAP prevention.

This placebo controlled trial was methodologically rigorous because it was adequately powered, randomised, blinded, and concealed; had explicit inclusion and exclusion criteria and outcomes; and had complete follow up for the primary end point. It also had standard protocols regarding head elevation to minimise confounders.

In this trial, besides the treatment, normal tooth brushing and saline cleansing of the oral cavity were administered to all patients. * Combining oral hygiene with $\mathrm{CHX}$ decontamination may have enhanced the removal of oral micro-organisms. Additionally, the concentration of $\mathrm{CHX}(2 \%)$ and the site (buccal cavity) and frequency of application ( 4 times/d) might be important considerations as another well designed trial differing in these aspects failed to find $\mathrm{CHX}$ effective.

Based on the trial by Koeman et al, $\mathrm{CHX}$ decontamination is promising for patients mechanically ventilated for $\geqslant 48$ hours, although its routine use for all ICU patients should probably await studies on the acquisition of long term resistance. This strategy to modulate oropharyngeal colonisation is simple, inexpensive, and minimally labour intensive. Its use should complement rather than replace basic oral hygiene, an integral aspect of nursing. Other strategies such as semirecumbent positioning should also be used whenever possible.

Ee Yuee Chan, RN, MN Tan Tock Seng Hospital Singapore

*Information provided by author

1 Fourrier F, Dubois D, Pronnier $P$, et al. Effect of gingival and dental plaque antiseptic decontamination on nosocomial infections acquired in the intensive care unit: a double-blind placebo-controlled multicenter study. Crit Care Med 2005; 33:1728-35.

Chlorhexidine $(\mathrm{CHX})$ or $\mathrm{CHX}$ plus colistin $(\mathrm{COL})$ v placebo in mechanically ventilated patients ${ }^{*}$

\begin{tabular}{llllll}
\hline Outcomet & CHX & CHX + COL & Placebo & RRR (95\% CI) & NNT (CI) \\
\hline Ventilator associated pneumonia & $10 \%$ & - & $18 \%$ & $63 \%(19$ to 83$)$ & 10 (7 to 30$)$ \\
& - & $13 \%$ & $18 \%$ & $52 \%(7$ to 76$)$ & 11 (8 to 83) \\
\hline
\end{tabular}

*Abbreviations defined in glossary; RRR, NNT, and $\mathrm{Cl}$ calculated from control event rates and hazard ratios in article.

tFollow up to diagnosis of ventilator associated pneumonia, death, or extubation. 